\title{
Analysis of Histological and Immunohistochemical Patterns of the Liver in Posthepatitic and Alcoholic Cirrhosis by Computerized Morphometry
}

\author{
Laura Vizzotto, Maurizio Vertemati, Marcello Gambacorta, Guido Sabatella, Vanda Spina, \\ Ernesto Minola \\ Department of Human Anatomy, Faculty of Medicine (LV, MV, GS, VS); and Department of Pathology \\ (MG, EM), Niguarda Hospital, Milan, Italy
}

To assess the degree of fibrosis and the structural changes affecting parenchymal and extraparenchymal components in liver cirrhosis, a computerized morphometric model has been applied to liver specimens from patients with posthepatitic and alcoholic cirrhosis. All specimens have been stained with chromotrope-aniline blue method and monoclonal antibodies against cytokeratin 7, CD31, and VIII factor. Volume fractions of parenchymal compartment and fibrosis have been determined stereologically on CAB slices; moreover, volume fractions of portal bile ducts and proliferated bile ductules, hepatocytes with biliary metaplasia, capillary units, and vascular structures have been measured. Volume fraction of fibrosis was higher in alcoholic cirrhosis when compared with the case of posthepatitic cirrhosis. Volume fractions describing parenchymal compartment showed a similar trend in both viral groups. The main differences were related to immunohistochemical stainings. Volume fraction of hepatocytes with biliary metaplasia was higher in hepatitis $\mathrm{C}$ virus-related cirrhosis, whereas volume fractions of biliary structures were more prominent in hepatitis B virus-related cirrhosis. Capillary units were more prominent in posthepatitic cirrhosis than in alcoholic cirrhosis. Interestingly, both forms of posthepatitic cirrhosis show similar features when compared with alcoholic cirrhosis. Our computerized morphometric model well describes and quantifies the morphological alterations of the liver, and it could represent an adjunctive tool to evaluate the degree of dysplastic phenomena involving parenchymal and extraparenchymal components.

Copyright () 2002 by The United States and Canadian Academy of Pathology, Inc

VOL. 15, NO. 8, P. 798, 2002 Printed in the U.S.A.

Date of acceptance: May 10, 2002.

Address reprint requests to: Laura Vizzotto, Dipartimento di Anatomia Umana-Università degli Studi di Milano, Via Mangiagalli 31, $20133 \mathrm{Mi}$ lano, Italy; e-mail: laura.vizzotto@unimi.it; fax: 0039-02-38210298.

DOI: 10.1097/01.MP.0000024365.92937.5E
KEY WORDS: Cirrhosis, Image analysis, Immunohistochemistry, Liver, Morphometry.

Mod Pathol 2002;15(8):798-806

Liver cirrhosis is the common manifestation of chronic viral hepatitis, chronic autoimmune hepatitis, and alcoholic liver disease, according to a geographical distribution depending on the different etiologic factors (1).

Cirrhosis is morphologically characterized by structural alterations of the liver parenchyma and microcirculation as a consequence of increased production and deposition of collagens $(2,3)$. Normal liver cells are damaged and replaced by scar tissue, decreasing the amount of normal liver tissue; the loss of normal liver tissue leads to hepatic failure. The distortion of the normal liver structure by the scar tissue leads to nodular changes that could interfere with the flow of blood through the liver and could contribute to the development of portal hypertension in cirrhosis (4). However, other authors have suggested that other factors, such as hepatocyte swelling and collagen in Disse space could be involved in the development of portal hypertension (5).

Moreover, hepatocellular carcinoma is a frequent complication of cirrhosis from many etiologies, as a consequence of regenerative and dysplastic processes that involve an abnormal vascularization $(6,7)$.

Quantitative investigation of the degree of fibrosis offers a clue for evaluation of the morphologic pattern and the progression of the inflammatory process and for the clinical staging of the patients $(8,9)$.

The most reliable method to evaluate these alterations is liver biopsy (10). Different methods of semiquantitative analysis have been applied to evaluate the degree of fibrosis $(11,12)$. In our study, we developed a computer-aided morphometric model to describe and quantify the architectural 
changes in the cirrhotic liver by traditional histological techniques and immunohistochemistry.

We have applied our computerized morphometric model to different subsets of patients affected by viral and alcoholic cirrhosis to evaluate parenchymal, sinusoidal, and/or biliary variables that could provide a more detailed differential morphological description of pathological changes affecting cirrhotic liver.

\section{PATIENTS AND METHODS}

We randomly selected thirty-three surgically removed livers from 33 patients ( 23 men, 10 women) who underwent orthotopic liver transplantation (OLT) at Niguarda Hospital, Milan. The etiology of liver cirrhosis was respectively hepatitis B virus (HBV)-related in 9 patients, hepatitis $\mathrm{C}$ virus (HCV)-related in 11 patients, and alcoholic in 13 patients. Diagnosis of cirrhosis was based on standard serological assays, abnormal liver function tests, and liver biopsy. The mean duration of the cirrhosis before transplantation was 15 months (range, 14-17 mo). Patients with a diagnosis of alcoholic cirrhosis have been abstinent for a period of 6 months before transplantation.

In patients affected by HCV-related cirrhosis, serum detection of HCV RNA has been performed by reverse transcription polymerase chain reaction (RT-PCR); in patients affected by HBV-related cirrhosis, quantitative assessment of HBV DNA levels has been performed by branched DNA assay (bDNA). In this regard, all patients affected by posthepatitic cirrhosis showed significant serum levels of HCV RNA and HBV DNA, respectively, in HCVand HBV-related cirrhosis. We excluded cirrhotic livers affected by hepatocellular carcinoma.

Patients' clinical data according to etiology of cirrhosis are listed in Table 1.

Some $1-\mathrm{cm}^{3}$ blocks have been obtained from the VIII hepatic segment after liver recipient hepatectomy, then immediately fixed in $10 \%$ formalin and embedded in paraffin. For each case, only one block has been randomly selected. From the selected block, four consecutive sections $4 \mu \mathrm{m}$ in thickness have been obtained. For each block, one section has been stained with hematoxylin and eosin to confirm the diagnosis of liver cirrhosis.

To reduce as far as possible the variability between different stains in each case, four serial consecutive sections have been selected.

For each section series, different staining methods have been applied:

- Chromotrope-aniline blue method (CAB trichromic assay) to assess the degree of fibrosis and the size of cirrhotic nodules (13).

- Monoclonal antibodies against cytokeratin 7 (CK7). CK7 is a member of a group of waterinsoluble proteins that form monofilaments in the cytoskeleton of most epithelial tissues. CK7 is a basic cytokeratin that is found in most glandular epithelia and in transitional epithelia. Epithelium in the lung and breast are positive, whereas colon and prostate epithelial cells are negative. In normal liver, hepatocytes are negative, whereas bile duct epithelium is positive $(14,15)$.

- Monoclonal antibodies against CD31, a specific and sensitive marker to detect capillary units that also stains arterial and venous endothelium. Capillary units have been defined as CD31-immunoreactive microvessels (16).

- Monoclonal antibodies against VIII factor (FVIII) to detect large arterial and venous vessels. The antigen related to VIII factor is detectable inside the cytoplasm of the cells that line the lumen of all blood vessels, and therefore, it really is a sensitive marker for identifying endothelial cells (17).

The morphometric analysis has been performed at two magnification levels by using in interactive approach a high-resolution computerized image analyzer (Kontron-Zeiss KS300). The image analyzer included a light microscope with a color video camera (JVC TK-C1381EG), a Pentium 200-MHz IBM-compatible computer (RAM, 64 megabytes). The software system, tailored to the research needs of our team, consisted of different programs to control interactively the scanning stage and auto-focus functions.

TABLE 1. Clinical and Biochemical Data of the Patients, Ranges (Means)

\begin{tabular}{|c|c|c|c|}
\hline Characteristic & $\mathrm{HCV}$ & $\mathrm{HBV}$ & Alcoholic \\
\hline Ratio male-female (M:F) & $8: 3$ & $6: 3$ & $9: 4$ \\
\hline \multirow[t]{2}{*}{ Age of patients } & M 33-53 (47.4) & M 36-59 (48.7) & M 35-59 (48.5) \\
\hline & F $47-57(50.4)$ & F 46-56 (52.0) & F 48-59 (53.8) \\
\hline Serum total bilirubin, $\mathrm{mg} / 100 \mathrm{~mL}$ & $0.9-3.9(2.5)$ & $0.7-4.2(2.8)$ & $0.8-4.1(2.8)$ \\
\hline Alkaline phosphatase, IU/L & $108-152(144)$ & $112-179(149)$ & $110-165$ (139) \\
\hline Prothrombin time, \% & $41-62(48)$ & $43-62(50)$ & $45-65(52)$ \\
\hline Platelets, $\times 1000 / \mathrm{mL}$ & $82-103(89)$ & $84-100(92)$ & $83-111(95)$ \\
\hline Alanine transpherase, IU/L & 73-114 (72) & $70-120(76)$ & $68-110(68)$ \\
\hline Child-Pugh score, $\mathrm{N}^{\circ}$ of patients/score & $6 / \mathrm{C} 5 / \mathrm{B}$ & $5 / \mathrm{C} 4 / \mathrm{B}$ & $7 / \mathrm{C} 6 / \mathrm{B}$ \\
\hline
\end{tabular}

M, male; F, female. 
Images acquired by video camera are displayed on the monitor. The analyzer automatically superimposes to each microscopic field different grids of points, included in a test area of $504 \times 504$ pixels, allowing an evaluation of the stereological variables (18). The observer can interactively apply techniques of enhancement for a better definition of parenchymal and vascular structures. It is also possible to exclude fields too damaged to be analyzed. An algorithm automatically controls the scanning stage operation to avoid duplicate measurements of the same structures. This way, an unbiased sampling is assured.

On CAB-stained slices at $2.5 \times$ magnification, the size distribution and the number of cirrhotic nodules in unit volume were calculated. The image size of the microscopic field displayed on the monitor (standard resolution, $1024 \times 768$ pixels) was about 80 times greater than the effective size of the sample. Because the size of nodules was always greater than the displayed microscopic field, a meander scan area corresponding to three contiguous fields in the $\mathrm{X}$ and $\mathrm{Y}$ directions $(3 \times 3)$ was determined and automatically created by the computer.

The profile of each nodule entirely included in this scan area displayed on the monitor was manually outlined.

Moreover, volume fractions of parenchyma and nonparenchyma (fibrosis and portal triads) have been determined. These values represent reference volumes for parenchymal and extraparenchymal components evaluated at higher magnification.

Three-dimensional size distribution and mean diameter of cirrhotic nodules were derived by diameter analysis according to the Schwartz-Saltykov method (19), modified after De Hoff (20). The area values were collected in a database for statistical analysis.

On CAB-stained slices at $40 \times$, volume fractions of hepatocytes (nuclei and cytoplasm), sinusoids, fibrosis, centrilobular veins, portal triads, and related structures (i.e., arteries, veins, bile ducts) were calculated. The overall magnification results from objective $\times$ optovar factor $\times$ digital videocamera factor $(40 \times 1.2 \times 2.6)$. As a consequence, the side of each microscopic field is $\cong 79.5 \mu \mathrm{m}$. A total of 200 microscopic fields, systematically selected, was examined for each case, so to make a complete scanning of the sample.

For each case, the analyzed area therefore corresponded to $\sim 1.3 \mathrm{~mm}^{2}$. This area is considered a representative sample of the human liver (21).

On immunostained sections at $40 \times$ magnification, volume fractions of the different structures were calculated. Volumetric analysis was performed by differential point counting (22).

\section{Morphometric Model}

The adopted model requires the determination of the following variables $(23,24,25)$ :

1) CAB method:

a) Low magnification

Volume fractions (referred to the entire liver) occupied by:

- Parenchyma $\left(\mathrm{V}_{\mathrm{v}} \mathrm{par}\right)$

- Extraparenchyma $\left(\mathrm{V}_{\mathrm{v}}\right.$ extra)

- Fibrosis $\left(\mathrm{V}_{\mathrm{v}}\right.$ fib)

- Portal triads $\left(\mathrm{V}_{\mathrm{v}} \mathrm{vasc}\right)$

- Mean cirrhotic nodules diameter $\left(\overline{\mathrm{d}}_{\mathrm{cir}}\right)$

- Observed number of cirrhotic nodules $(\mathrm{N})$

b) High magnification

Volume fractions (referred to the entire liver) occupied by:

- Hepatocyte nuclei $\left(\mathrm{V}_{\mathrm{v}}\right.$ nuc $)$

- Centrilobular veins $\left(\mathrm{V}_{\mathrm{v}}\right.$ cenlo)

- Lumen and endothelium of sinusoids $\left(\mathrm{V}_{\mathrm{v}}\right.$ sinend)

- Fibrosis $\left(\mathrm{V}_{\mathrm{v}}\right.$ fibros $)$

- Portal triads $\left(\mathrm{V}_{\mathrm{v}}\right.$ tria):

- Lumen and endothelium of hepatic arteries ( $\mathrm{V}_{\mathrm{v}}$ arter)

- Lumen and endothelium of portal veins ( $\mathrm{V}_{\mathrm{v}}$ veins)

- Bile ducts $\left(\mathrm{V}_{\mathrm{v}}\right.$ ducts $)$

2) Immunǒstăining

Volume fractions occupied by:

- Capillary units $\left(\mathrm{V}_{\mathrm{v}} \mathrm{cpu}\right)$, referred to liver parenchyma $\rightarrow$ CD31

- Endothelium of hepatic arteries $\left(\mathrm{V}_{\mathrm{v}}\right.$ endar), referred to fibrosis $\rightarrow$ FVIII

- Endothelium of hepatic veins $\left(\mathrm{V}_{\mathrm{v}}\right.$ endve $)$, referred to fibrosis $\rightarrow$ FVIII

- Lumen of hepatic arteries ( $\mathrm{V}_{\mathrm{v}}$ lumar), referred to fibrosis $\rightarrow$ FVIII

- Lumen of portal veins $\left(\mathrm{V}_{\mathrm{v}}\right.$ lumve), referred to fibrosis $\rightarrow$ FVIII

- Bile ducts $\left(\mathrm{V}_{\mathrm{v}}\right.$ bilduc), referred to the entire liver $\rightarrow$ CK7

- Hepatocytes with biliary metaplasia $\left(\mathrm{V}_{\mathrm{v}}\right.$ hepbil), referred to liver parenchyma $\rightarrow$ CK7

\section{Statistical Analysis}

Pentium III MHz computer (RAM 128 megabytes) and SPSS, Version 9.0 software were used for statistical analysis. For each parameter, a comparison among different etiologies has been performed by variance analysis followed by Scheffé posthoc test $(26,27)$. 


\section{RESULTS}

Table 2 shows the main morphometric variables measured on $\mathrm{CAB}$ staining for the different groups of etiologies. All results are given as mean \pm SD. The mean diameter of cirrhotic nodules is higher in $\mathrm{HBV}$-related cirrhosis when compared with HCV

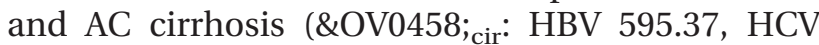
446.78, AC $421.61 \mu \mathrm{m})$

Data related to volume fractions:

- At low magnification, volume fraction of fibrosis is higher in the alcoholic group, while both viral groups have similar values $\left(\mathrm{V}_{\mathrm{v}}\right.$ fib: $\mathrm{HCV}$ .3027, HBV .3187, AC .3769). This data has been confirmed at higher magnification.

- At low magnification, volume fraction of parenchyma shows an opposite trend when compared to $\mathrm{V}_{\mathrm{v}}$ fib $\left(\mathrm{V}_{\mathrm{v}}\right.$ par: HCV .6348, HBV .6439, AC .5800).

- At low magnification, volume fraction of vascular compartment shows a significant difference between viral forms ( $\mathrm{V}_{\mathrm{v}}$ vasc: HCV .0625, HBV .0375, AC .0431).

- At high magnification, volume fractions describing parenchymal compartment show that both viral groups have a similar trend when compared to alcoholic group $\left(\mathrm{V}_{\mathrm{v}}\right.$ cel: $\mathrm{HCV}$ .5043, HBV .5033, AC .4109; $\mathrm{V}_{\mathrm{v}}$ sinend: HCV .1231 , HBV .1340, AC .1564; V $\mathrm{v}_{\mathrm{v}}$ cenlo: HCV .0063, HBV .0056, AC .0199); meanwhile, volume fraction of hepatocyte nuclei is characterized by a similar distribution among the three aetiologic groups $\left(\mathrm{V}_{\mathrm{v}}\right.$ nuc: $\mathrm{HCV} .0290$, HBV .0236, AC .0283).

Figures 1 and 2 illustrate the distribution of the parenchymal and extraparenchymal compartments relative to the entire liver. Moreover, the percentage distribution of the subcomponents of both parenchymal and extraparenchymal compartments is shown.

Figure 3 compares the size distribution of the cirrhotic nodules according to the etiology. This figure well describes the more "regular" distribution of the diameter classes of the nodules in alcoholic cirrhosis when compared with posthepatitic cirrhosis.

Table 3 shows the morphometric variables evaluated on the different immunostains (i.e., FVIII, CyK7, CD31):

- The volume fraction of CD31 capillary units is significantly higher in HBV-related cirrhosis when compared to postalcoholic cirrhosis ( $\mathrm{V}_{\mathrm{v}} \mathrm{cpu}$ : HCV .0256, HBV .0378, AC .0175);

- The most striking result is related to variables evaluated on CyK7; volume fraction of portal bile ducts and proliferated bile ductules is significantly higher in $\mathrm{HBV}$-related cirrhosis when compared to HCV-related cirrhosis $\left(\mathrm{V}_{\mathrm{v}}\right.$ bilduc: HCV .0282, HBV .0581, AC .0344);

In contrast, the volume fraction of biliarized hepatocytes shows an opposite trend: in fact, significantly higher values characterize HCVrelated cirrhosis when compared to both HBVrelated cirrhosis and postalcoholic cirrhosis (V $\mathrm{v}_{\mathrm{v}}$ hepbil: HCV .0875, HBV .0306, AC .0259).

\section{DISCUSSION}

Liver cirrhosis is characterized by hepatocellular, fibrotic, and microvascular changes that cause morphological alterations of the hepatic architecture, leading to functional derangement of the hepatic function (4). The histopathology of hepatic

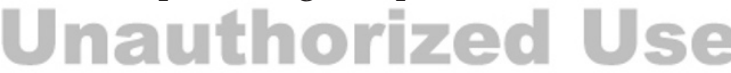

TABLE 2. Main Morphometric Variables Evaluated for Etiology (Means \pm Standard Deviations)

\begin{tabular}{|c|c|c|c|c|c|}
\hline Variables & Unit of Measure & HCV Cirrhosis & Alcoholic Cirrhosis & HBV Cirrhosis & $P$ Values $^{a}$ \\
\hline \multicolumn{6}{|l|}{ Low magnification } \\
\hline $\mathrm{V}_{\mathrm{v}}$ par & $\mathrm{mm}^{3} / \mathrm{mm}^{3}$ & $0.6348 \pm 0.1013$ & $0.5800 \pm 0.1217$ & $0.6439 \pm 0.0711$ & .305 \\
\hline $\mathrm{V}_{\mathrm{v}}$ extra & $\mathrm{mm}^{3} / \mathrm{mm}^{3}$ & $0.3652 \pm 0.1013$ & $0.4200 \pm 0.1217$ & $0.3561 \pm 0.0711$ & .305 \\
\hline $\mathrm{V}_{\mathrm{v}} \mathrm{fib}$ & $\mathrm{mm}^{3} / \mathrm{mm}^{3}$ & $0.3027 \pm 0.0907$ & $0.3769 \pm 0.1059$ & $0.3187 \pm 0.0668$ & .138 \\
\hline $\mathrm{V}_{\mathrm{v}}$ vasc & $\mathrm{mm}^{3} / \mathrm{mm}^{3}$ & $0.0625 \pm 0.0227^{\circ}$ & $0.0431 \pm 0.0218^{\circ *}$ & $0.0374 \pm 0.0136^{*}$ & .025 \\
\hline No. of cirrhotic nodules $(N)$ & - & 210 & 331 & 98 & \\
\hline$\overline{\mathrm{d}}_{\mathrm{cir}}$ & $\mu \mathrm{m}$ & $446.78^{\circ}$ & $421.61^{\circ}$ & $595.37^{*}$ & .018 \\
\hline \multicolumn{6}{|l|}{ High magnification } \\
\hline $\mathrm{V}_{\mathrm{v}}$ cel & $\mathrm{mm}^{3} / \mathrm{mm}^{3}$ & $0.5043 \pm 0.0942^{\circ}$ & $0.4109 \pm 0.0932^{*}$ & $0.5033 \pm 0.0626^{\circ}$ & .037 \\
\hline $\mathrm{V}_{\mathrm{v}}$ nuc & $\mathrm{mm}^{3} / \mathrm{mm}^{3}$ & $0.0291 \pm 0.0069$ & $0.0283 \pm 0.0070$ & $0.0236 \pm 0.0070$ & .412 \\
\hline $\mathrm{V}_{\mathrm{v}}$ sinend & $\mathrm{mm}^{3} / \mathrm{mm}^{3}$ & $0.1231 \pm 0.0298$ & $0.1564 \pm 0.0709$ & $0.1340 \pm 0.0272$ & 0.342 \\
\hline $\mathrm{V}_{\mathrm{v}}$ cenlo & $\mathrm{mm}^{3} / \mathrm{mm}^{3}$ & $0.0063 \pm 0.0072$ & $0.0119 \pm 0.0146$ & $0.0065 \pm 0.0120$ & .503 \\
\hline $\mathrm{V}_{\mathrm{v}}$ fibros & $\mathrm{mm}^{3} / \mathrm{mm}^{3}$ & $0.3326 \pm 0.0890$ & $0.3800 \pm 0.1000$ & $0.3298 \pm 0.0692$ & .082 \\
\hline \multicolumn{6}{|l|}{$\mathrm{V}_{\mathrm{v}}$ tria } \\
\hline $\mathrm{V}_{\mathrm{v}}$ ducts & $\mathrm{mm}^{3} / \mathrm{mm}^{3}$ & $0.0122 \pm 0.0069^{\circ}$ & $0.0142 \pm 0.0093^{\circ}$ & $0.0094 \pm 0.0055^{*}$ & .036 \\
\hline $\mathrm{V}_{\mathrm{v}}$ arter & $\mathrm{mm}^{3} / \mathrm{mm}^{3}$ & $0.0010 \pm 0.0016$ & $0.0025 \pm 0.0032$ & $0.0006 \pm 0.0008$ & .631 \\
\hline $\mathrm{V}_{\mathrm{v}}$ veins & $\mathrm{mm}^{3} / \mathrm{mm}^{3}$ & $0.0205 \pm 0.0119$ & $0.0241 \pm 0.0148$ & $0.0164 \pm 0.0118$ & .489 \\
\hline
\end{tabular}

Volume fractions refer to the entire liver. Morphometric variables were described in Patients and Methods. Superscript symbols indicate homogeneous subsets in Schaffe's post hoc test; data subsets marked with the same symbol were homogeneous.

$\mathrm{HCV}$, hepatitis C virus-related; HBV, hepatitis B virus-related.

${ }^{a}$ Probability values from ANOVA (source between pathologies). 
Alcoholic cirrhosis

$100 \%$
HBV cirrhosis

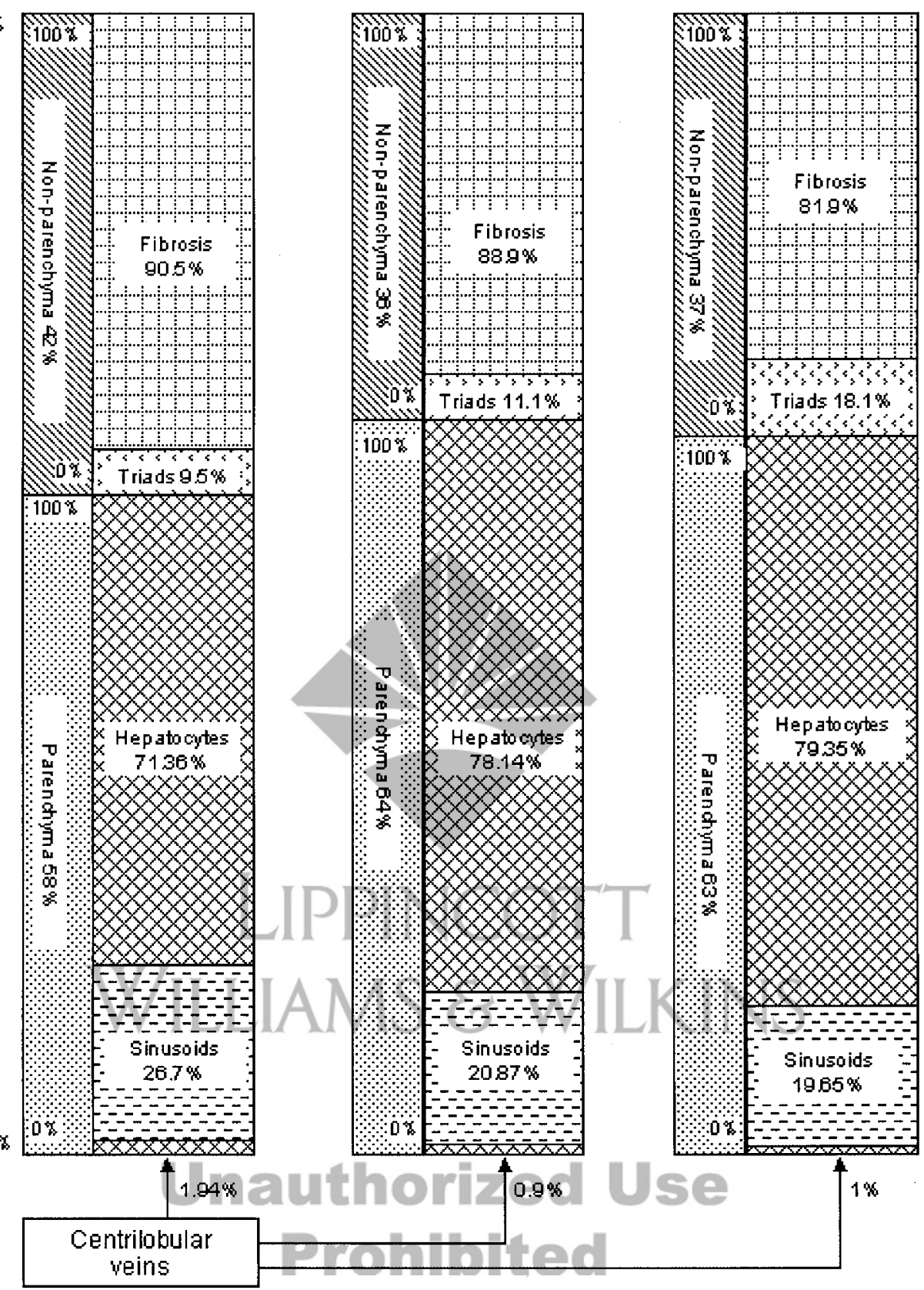

FIGURE 1. Parenchymal and extraparenchymal volume fractions in the whole liver: comparison among different etiologies on CAB trichromic assay. The percentage distribution of the subcomponents related to both compartments is also shown.

cirrhosis can resemble different patterns depending on the stage of disease progression $(28,29)$.

Another important point that needs to be stressed is the role of the etiology of the cirrhotic process. Viral (i.e., HBV or HCV related) and not viral (i.e., alcoholic) cirrhosis can differ not only with regard to the clinical outcome but also with a different histological pattern, resembling the influence of a persisting viral replication that in virusrelated cirrhosis can promote a continuous fibrogenic process.

Moreover, the structural alterations affecting lobular architecture and the regenerative activity of hepatocytes have been considered to play a key role for the occurrence of hepatocellular carcinoma (30).

In our study, we have developed a morphometric model to investigate the parenchymal and extraparenchymal morphology of alcoholic cirrhosis and posthepatitic cirrhosis (HBV and HCV related). In this regard, we have applied both colorimetric staining by $\mathrm{CAB}$ trichrome, to evaluate the degree of fibrosis and architectural alterations involving liver parenchyma, and immunostainings to better identify and quantify the alterations related to vascular and biliary structures. 
Alcoholic cirrhosis

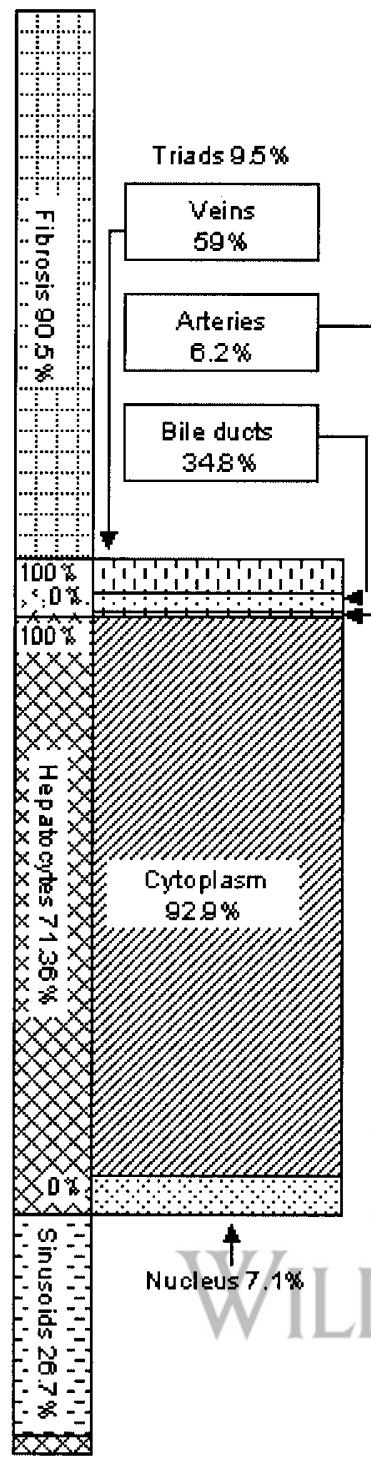

HBV cirrhosis

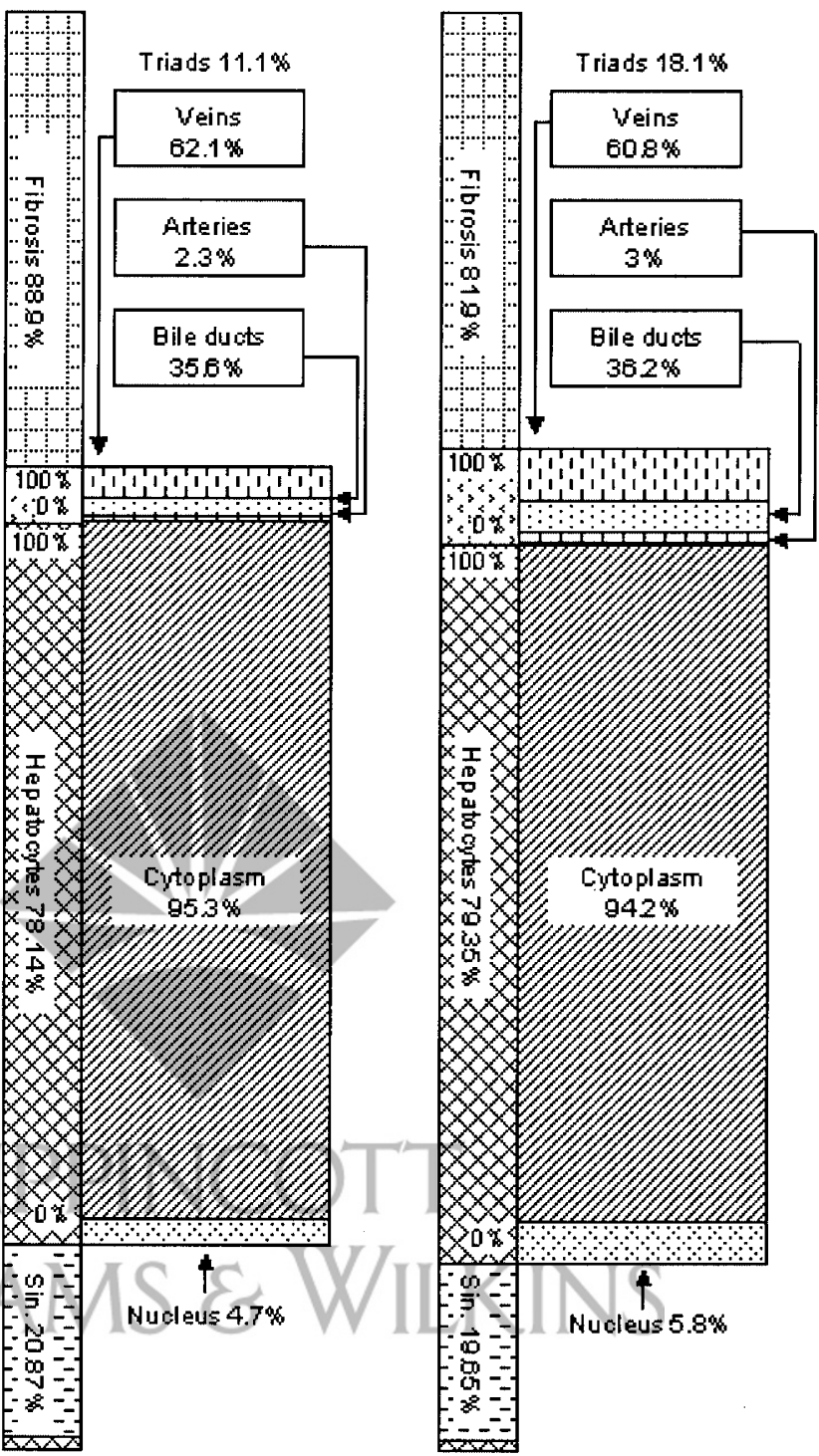

FIGURE 2. Further parenchymal and extraparenchymal compartments. Hepatocytes and triad volume fractions among different etiologies on CAB trichromic assay. The percentage distribution of the subcomponents related to both compartments is also shown.

The morphometric analysis on $\mathrm{CAB}$ trichrome stains showed that HBV-related cirrhosis is characterized by a higher mean diameter of cirrhotic nodules when compared with HCV and AC cirrhosis (Table 2). Moreover, AC cirrhosis displays a more uniform distribution of the diameter classes of the cirrhotic nodules when compared with posthepatitic cirrhosis (Fig. 3).

At low magnification, although AC shows a higher volume fraction of fibrosis when compared with posthepatitic cirrhosis, no statistical difference among etiologies has been found. This result has been confirmed at high magnification.

Previous studies $(11,12)$ have reported that the area of fibrosis (ratio of the collagen fibers to the area of entire liver tissue specimen) was significantly higher in hepatitis $\mathrm{C}$ patients than in hepa- titis B patients as a consequence of the persistence of both necroinflammatory and virus-induced fibrotic reaction. These data correlated to the period of evolution from HCV-related chronic hepatitis to cirrhosis. The authors also showed that the histological characteristics of HCV-related cirrhosis are septal fibrosis with small regenerative nodules and moderate inflammatory reaction.

In this regard, all of our patients affected by HBVrelated cirrhosis had detectable serum levels of HBV DNA (bDNA assay). HBV presence and activity could result in sustained fibrogenic process, and these data can explain, at least in part, the apparent discrepancy between reported data and our results.

Morphometric variables describing parenchymal compartment show peculiar differences among alcoholic and posthepatitic cirrhosis (Figs. 1 and 2). 

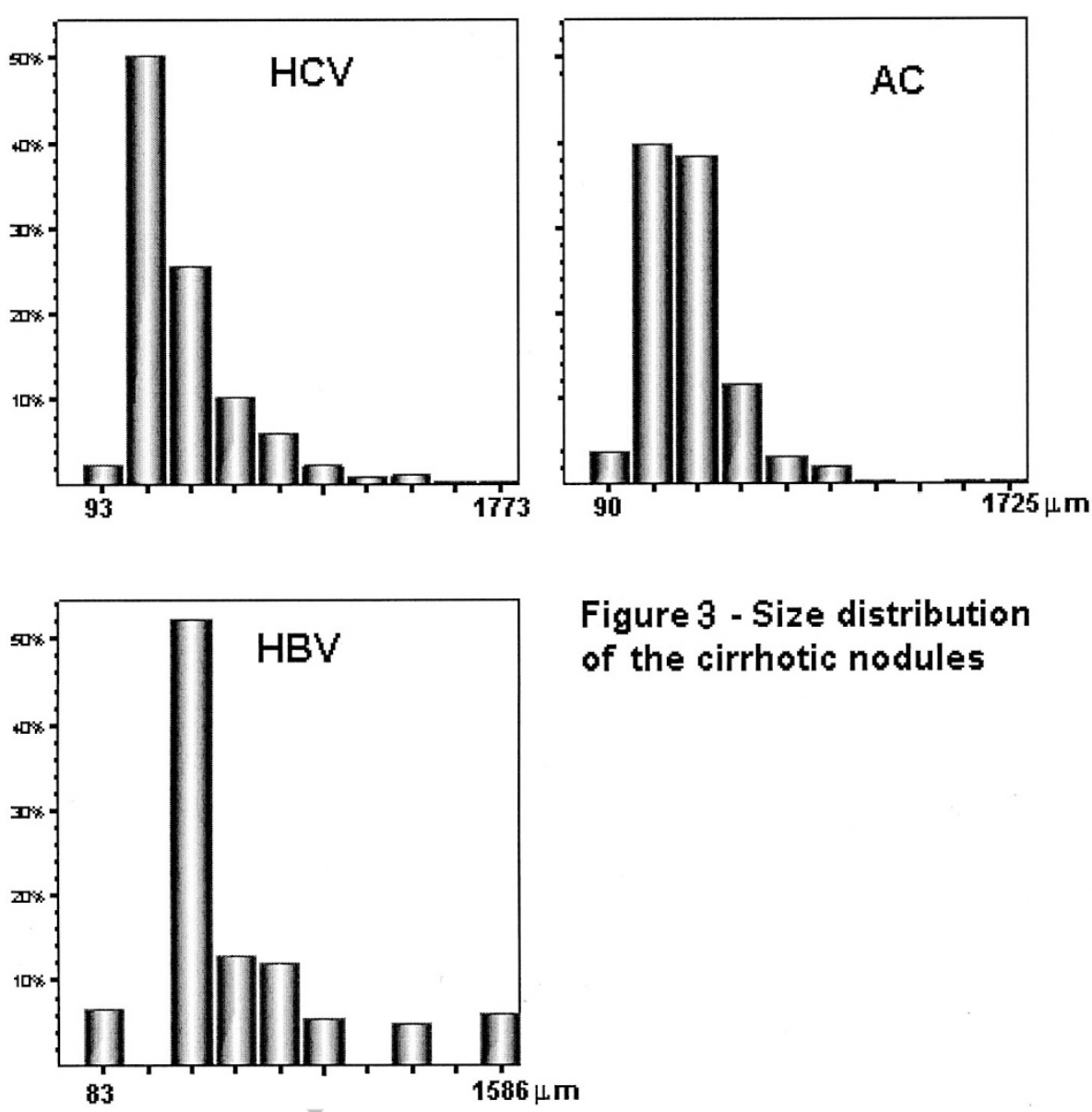

\section{Figure 3 - Size distribution of the cirrhotic nodules}

FIGURE 3. Size distribution of the cirrhotic nodules according to the different etiologies. Alcoholic cirrhosis displays a more regular distribution of the diameter classes of the nodules.

TABLE 3. Morphometric Variables Evaluated by Immunostaining (Means \pm Standard Deviations)

\begin{tabular}{|c|c|c|c|c|c|}
\hline Variable & Marker & HCV Cirrhosis & $\begin{array}{l}\text { Alcoholic } \\
\text { Cirrhosis }\end{array}$ & HBV Cirrhosis & $P$ Values ${ }^{a}$ \\
\hline $\mathrm{V}_{\mathrm{v}}$ endar & FVIII & $0.0022 \pm 0.002$ & $0.0007 \pm 0.0006$ & $0.0022 \pm 0.003$ & 0.121 \\
\hline $\mathrm{V}_{\mathrm{v}}$ endve & FVIII & $0.0117 \pm 0.005^{*}$ & $0.0047 \pm 0.002^{\circ}$ & $0.0081 \pm 0.004^{* o}$ & 0.001 \\
\hline$V_{\mathrm{v}}$ lumar & FVIII & $0.0075 \pm 0.009$ & $0.0040 \pm 0.005$ & $0.0034 \pm 0.004$ & 0.346 \\
\hline $\mathrm{V}_{\mathrm{v}}$ lumve & FVIII & $0.0603 \pm 0.027$ & $0.0428 \pm 0.034$ & $0.0450 \pm 0.039$ & 0.413 \\
\hline $\mathrm{V}_{\mathrm{v}} \mathrm{cpu}$ & CD31 & $0.0256 \pm 0.005^{\circ *}$ & $0.0175 \pm 0.007^{\circ}$ & $0.0378 \pm 0.028^{*}$ & 0.019 \\
\hline $\mathrm{V}_{\mathrm{v}}$ bilduc & CyK-7 & $0.0282 \pm 0.087^{\circ}$ & $0.0344 \pm 0.015^{\mathrm{o} *}$ & $0.0581 \pm 0.037^{*}$ & 0.014 \\
\hline $\mathrm{V}_{\mathrm{v}}$ hepbil & CyK-7 & $0.0875 \pm 0.047^{*}$ & $0.0259 \pm 0.020^{\circ}$ & $0.0305 \pm 0.012^{\circ}$ & $<0.001$ \\
\hline
\end{tabular}

See Patients and Methods section for description of morphometric variables. Superscript symbols indicate homogeneous subsets in Scheffé post hoc test; data subsets marked with the same symbol were homogeneous. HCV, hepatitis C virus-related; HBV, hepatitis B virus-related.

${ }^{a}$ Probability values from ANOVA (source between pathologies).

Volume fraction occupied by hepatocytes is lower in AC cirrhosis; moreover, HBV- and HCV-related cirrhosis show similar results. In contrast, volume fraction of sinusoids shows opposite results. With regard to extraparenchymal components, no significant difference has been found among groups; but it should be noted that both HBV- and HCV-related cirrhosis show the same trend (Table 2).

The morphometric analysis of immunostained specimens has evidenced some characteristic patterns of the different forms of cirrhosis (Table 3).

The more interesting results are concerned with monoclonal antibodies against CK7. It is well known that CK7 is, in normal liver, restricted to bile duct cells. CK7 stains ducts and metaplastic cells that are not destroyed by inflammation in chronic active hepatitis.

Irrespective of the etiology, after a biliary obstruction, a variable number of hepatocytes at the periphery of the hepatic lobule express CK7 (15). Moreover, longstanding cholestasis increases the bile ductules in the portal areas (31). Interestingly, these CK7-positive hepatocytes maintain all morphological features of hepatocytes. These hepatocytes could be representative of a transitional cell stage, as a consequence of metaplastic phenomena 
involving both intrahepatic biliary epithelium and parenchymal hepatocytes.

The periportal ductal metaplasia has been related to the persistence of hepatitis B virus in cells deriving from biliary metaplasia of infected hepatocytes (32). It has been shown that both almost all proliferated bile ductules and some hepatocytes, especially those adjacent to bile ductules, show an altered expression of blood group antigens in any of the hepatobiliary diseases (33).

In our study, morphometric analysis has evidenced a significantly higher volume fraction of CK7-positive hepatocytes in HCV-related cirrhosis. These cells are usually located at the periphery of hepatic lobules and near periportal fibrosis.

Hepatocellular carcinoma has been related to viral infections, heavy alcoholic liver disease, and hemochromatosis (34). It could be hypothesized that the assessment of biliary metaplasia can be an adjunctive tool to evaluate the degree of dysplastic phenomena involving the liver in posthepatitic cirrhosis.

CK7 stains epithelial cells in both normal and newly formed bile ducts. Our results show a higher volume fraction of bile ducts (i.e., portal bile ducts and proliferated bile ductules) in HBV-related cirrhosis. These data correlate with volume fractions of fibrosis and hepatocytes previously measured by $\mathrm{CAB}$ analysis, and they could be related to a continuous fibrogenic process induced by viral proliferation.

Volume fractions measured by CD31, a specific and sensitive marker for capillary, are higher in HBV-related cirrhosis. As recently suggested, an increase in the number of capillary units during liver cirrhosis can be a useful clue for differential diagnosis between nodules with low-grade and highgrade dysplasia and hepatocellular carcinoma, where the extension of capillarization is upregulated (35).

The analysis of volume fractions evaluated in FVIII-stained specimens did not show any significant difference between morphometric variables in the three groups, except volume fraction of the endothelium of hepatic veins. Although we have found slightly higher values in HCV group, the wide range of variability of these variables makes it difficult to come to any conclusion about these structures.

In conclusion, our morphometric model well describes the morphologic patterns of liver cirrhosis according to different etiologies. When considering parenchymal and fibrosis variables, both viralrelated cirrhoses show similar features when compared with AC. This trend is confirmed, at least in part, by immunostaining with monoclonal antibodies specifically directed against CK7 and CD31. HCV-related cirrhosis shows a higher volume frac- tion of CK7-positive hepatocytes, whereas ductal profiles are more represented in HBV-related cirrhosis. The number of capillary units, as documented by CD31, is higher in posthepatitic cirrhosis when compared with AC.

Computerized morphometry can be of value for quantifying the degree of liver fibrosis and both morphological and immunohistochemical alterations of parenchymal and extraparenchymal compartments in the cirrhotic liver that could be useful indicators in the histopathological assessment of the cirrhosis according to the different etiology.

\section{REFERENCES}

1. Anthony PP, Ishak KG, Nayak NC, Poulsen HE, Scheuer PJ, Sobin LH. The morphology of cirrhosis: definition, nomenclature and classification. Bull World Health Organ 1977; 55(4):521-40.

2. Friedman SL. The cellular basis of hepatic fibrosis: mechanisms and treatment strategies. N Engl J Med 1993;328:1828-35.

3. Burt AD. Cellular and molecular aspects of hepatic fibrosis. J Pathol 1993;170:105-14.

4. Popper H, Hutterer F. Hepatic fibrogenesis and disturbance of hepatic circulation. Ann NY Acad Sci 1970;170:88-99.

5. Orrego H, Blendis LM, Crossley IR, Blake JE, Medline A MacDonald A, et al. Correlation of intrahepatic pressure with collagen in the Disse space and hepatomegaly in humans and in the rat. Gastroenterology 1981;80:546-56.

6. Ferrell L, Wright T, Lake J, Roberts J, Asher N. Incidence and diagnostic features of dysplastic nodules vs small hepatocellular carcinoma in cirrhotic liver. Hepatology 1992;16:1372-81.

7. Libbrecht $/$, Graninx M, Nevens F, Desmet V, Roskams T. Predictive of liver cell dysplasia for development of hepatocellular carcinoma in patients with non-cirrhotic and cirrhotic viral hepatitis. Histopathology 2001;39(1):66-73.

8. Scheuer PJ. Classification of chronic viral hepatitis: a need for reassessment. J Hepatol 1991;13:372-4.

9. Desmet VJ, Gerber M, Hoofnagle JH, Manns M, Scheuer PJ. Classification of chronic hepatitis: diagnosis, grading and staging. Hepatology 1994;19:1513-20.

10. Friedman SL. Evaluation of fibrosis and hepatitis C. Am J Med 1999;107(6B):27S-30S.

11. Kage M, Shimamatsu K, Nakashima E, Kojiro M, Inoue O, Yano M. Long-term evolution of fibrosis from chronic hepatitis to cirrhosis in patients with hepatitis C: morphometric analysis of repeated biopsies. Hepatology 1997;25:1028-31.

12. Yano M, Kumada H, Kage M, Ikeda K, Shimamatsu K, Inoue $\mathrm{O}$, et al. The long-term pathological evolution of chronic hepatitis C. Hepatology 1996;23(6):1334-40.

13. Churg J, Prado A. A rapid Mallory trichrome stain (chromotrope-aniline blue). Arch Pathol 1956;62:505-6.

14. van Eyken P, Sciot R, Desmet VJ. A cytokeratin immunohistochemical study of cholestatic liver disease: evidence that hepatocytes can express "bile duct-type." Histopathology 1989;15(2):125-35.

15. James J, Lygidakis LJ, van Eyken P, Tanka AK, Bosch KS, Ramaekers FC, et al. Application of keratin immunocytochemistry and sirius red staining in evaluating intrahepatic changes with acute extrahepatic cholestasis due to hepatic duct carcinoma. Hepatogastroenterology 1989;36(3):151-5.

16. Schmidt D, von Hochstetter AR. The use of CD31 and collagen IV as vascular markers. A study of 56 vascular lesions. Pathol Res Pract 1995;191:410-4.

17. Roholl PJ, De Jong AS, Ramaekers FC. Application of markers in the diagnosis of soft tissue tumours. Histopathology 1985; 9(10):1019-35. 
18. Elias H, Henning A, Schwartz DE. Stereology: applications to biomedical research. Physiol Rev 1971;51:158-200.

19. Saltykov SA. In: Elias H, editor. A stereological method for measuring the specific surface area in metallic powders. Berlin: Springer-Verlag; 1967. p. 63-4.

20. De Hoff RT, Rhines FN, editors. Quantitative microscopy. New York: McGraw-Hill; 1968.

21. Weibel ER, Staubli W, Gnagi HR, Hess FA. Correlated morphometric and biochemical studies on the liver cell. J Cell Biol 1969;42:68-91.

22. Delesse A. Procédé méchanique pour déterminer la composition des roches. C R Acad Sci D 1847;51:544-67.

23. Oberholzer M, Rohr HP. Liver morphometry. In: Baak JPA, Oort J, editors. A manual of morphometry in diagnostic pathology. Berlin: Springer-Verlag; 1983. p. 126-31.

24. Vizzotto L, Romani F, Ferrario VF, Tommasini Degna C, Aseni P. Characterization by morphometric model of liver regeneration in the rat. Am J Anat 1989;185:444-54.

25. Vizzotto L, Vertemati M, Tommasini Degna C, Aseni P. Liver transplantation in man: morphometric analysis of the parenchymal alterations following cold ischemia and warm ischemia/reperfusion. J Anat 2001;198:603-10.

26. Armitage P. Regression and correlations. In: Armitage P, editor. Statistical methods in medical research. Oxford: Blackwell Scientific; 1973. p.147-66.

27. Snedecor GW, Cochran WG. Statistical methods. Ames, Iowa: Iowa State University Press; 1972.
28. Rahman SM, Itakura H, Motoda A. Regenerative pattern of liver cells in primary biliary cirrhosis, alcoholic cirrhosis, posthepatitic cirrhosis (HBV-related) and hepatocellular carcinoma: comparative analysis by computerised morphometry. Pathol Int 1996;46:267-73.

29. Ryoo JW, Buschmann RJ. Morphometry of liver parenchyma in needle biopsy specimens from patients with alcoholic liver disease: preliminary variables for diagnosis and prognosis of cirrhosis. Mod Pathol 1980;9(4):382-9.

30. Shimamatsu K, Kage M, Nakashima O, Kojiro M. Pathomorphological study of HCV anti-body-positive liver cirrhosis. J Gastroenterol Hepatol 1994;9:624-30.

31. Sirica AE. Ductular hepatocytes. Histol Histopathol 1995; 10(2):433-56.

32. Delladetsima JK, Vafiadis I, Tassopoulos NC, Kyriakou V, Apostolaki A, Smyrnoff T. HbcAg and HbsAg expression in ductular cells in chronic hepatitis B. Liver 1994;14(2):71-5.

33. Nakanuma Y, Sasaki M. Expression of blood group-related antigens in the intrahepatic biliary tree and hepatocytes in normal livers and various hepatobiliary diseases. Hepatology 1989;10(2):174-8.

34. Collier J, Sherman M. Screening for hepatocellular carcinoma. Hepatology 1998;27:273-8.

35. Roncalli M, Roz E, Coggi G, Di Rocco MG, Bossi P, Minola E, et al. The vascular profile of regenerative and dysplastic nodules of the cirrhotic liver: implications for diagnosis and classification. Hepatology 1999;30(5):1174-8.

\section{Book Review}

Gartner LP, Hiatt, JL: Electronic Slide Set for Color Textbook of Histology, 2nd Edition, Philadelphia, W.B. Saunders Company, 2001 (\$495.00).

This CD-ROM contains all 439 images used in the authors' corresponding text (i.e., Color Textbook of Histology, 2nd Edition) that are formatted in both standard JPEG (Joint Photographers Expert Group) and PDF (Portable Document Format). The main purpose of the program is to import the schematics as well as the light- and electron microscopic images into presentation programs (e.g., PowerPoint). The schematic images may be viewed as either labeled or unlabeled. The program is designed to run entirely from the CD, and an Internet browser is required to view its contents. Any version of Netscape may be used to run the program. Netscape Communicator 4.76 is included on the CD. The program's main menu screen makes finding desired image(s) easy. Images are initially displayed in a reduced size called thumbnails. The "Screen Version" button pulls up a larger version of the image that allows an image to be stored on a computer's hard drive in a standard JPEG format. Multiple images may be viewed at the same time. Adobe Acrobat Reader is needed to print images, and the CD comes with Acrobat Reader 4.0. In summary, this CD-ROM may be used for easy import of histology images into presentation programs, lecture handouts, and quizzes/ examinations. For lecture handouts and quizzes/ examinations, up to $10 \%$ of images may be used after obtaining a permission letter from the Rights and Permission's group of W.B. Saunders Company.

David L. McWhorter

The University of Health Sciences Kansas City, Missouri 